Sorda \& Sonora (1) 2018: 52 - 63

DOI: https://doi.org/10.18800/sordaysonora.201801.004

\title{
Sobre el estudio de la gramática: nota de cierre del curso de Estructura del Lenguaje
}

On the study of grammar: closing note of the Language Structure course

\author{
Jaime Vera Amorós \\ Pontificia Universidad Católica del Perú \\ jveraa@pucp.edu.pe
}

\section{RESUMEN}

En este texto pretendo (i) recoger y analizar los objetivos de un curso de lingüística para estudiantes de la facultad de Estudios Generales Letras de la Pontificia Universidad Católica del Perú; (ii) ofrecer algunas ideas personales sobre la lingüística como disciplina; y (iii) sintetizar las ideas y conceptos más importantes del curso.

Palabras clave: lingüística, gramática, enseñanza de la gramática, estudios gramaticales, estudios de las lenguas humanas

\section{AbSTRACT}

In this text I intend (i) to collect and analyze the objectives of a linguistics course for students of the Faculty of Estudios Generales Letras at the Pontifical Catholic University of Peru; (ii) offer some personal ideas about linguistics as a discipline; and (iii) synthesize the most important ideas and concepts of the course.

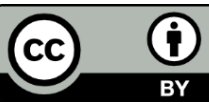


Keywords: linguistics, grammar, teaching grammar, grammar studies, study of human natural languages

\section{La lingüística ${ }^{1}$, y los cursos de Teoría General del Lenguaje y Es- tructura del Lenguaje ${ }^{2}$}

Existe una tradición milenaria de descripción de lenguas: los primeros en describir por escrito y sistemáticamente las reglas de su lengua fueron los gramáticos sánscritos y griegos. Los lingüistas de hoy son herederos de esa larga seguidilla de gramáticos (autores de gramáticas) y lexicógrafos (autores de diccionarios). Y, sin embargo, en los últimos cien años, la disciplina lingüística se ha expandido temáticamente hasta estudiar los más diversos aspectos del lenguaje humano y las lenguas. Por eso, al inicio les ofrecí una definición amplia, en los términos más abarcadores posibles, de lo que estudian los muy diversos lingüistas:

Una propuesta de definición abarcadora de la lingüística ${ }^{3}$

La lingüística es una disciplina que estudia de manera rigurosa y sistemática las diversas manifestaciones del lenguaje humano y de las lenguas humanas: tanto su estructura como su función; tanto en el presente como en la historia; tanto en relación con sus bases biológicas (genéticas y evolutivas) como con su desarrollo y uso individuales (neurológicos y cognitivos), y como con su papel en las relaciones sociales (en la interacción y en la estructura social). Es una disciplina extremadamente amplia en sus subramas (estudios gramaticales, psicolingüística, sociolingüística, lingüística histórica,

1 Este texto fue escrito originalmente para los estudiantes de una de las comisiones del curso Estructura del Lenguaje de la Pontificia Universidad Católica del Perú del ciclo 2016-2, de parte de uno de los jefes de prácticas del curso.

2 Estos son cursos dictados en la facultad de Estudios Generales Letras de nuestra universidad.

3 El texto de esta definición es de mi autoría, pero no dice nada nuevo ni especialmente original. Por una parte, muchos otros lingüistas ofrecen definiciones similares. Por otra, es cierto que muchos otros lingüistas ofrecen definiciones más restringidas y menos abarcadoras. 
etc.) como en sus enfoques teórico-explicativos (formalistas, funcionalistas, constructivistas, cognitivistas, etc.).

Una definición como esta incluye los muy diversos temas que se cubren en las muy distintas versiones del curso de Estructura del Lenguaje, Teoría General del Lenguaje, y Lenguaje y Sociedad (a la vez que habrá temas que no se tocan en ninguna de ellas) ${ }^{4}$. En esa especie de división del trabajo, al curso de Estructura le toca ser una presentación de una pequeña parte de la lingüística: el estudio de la gramática de las lenguas y, en particular, de la del castellano. A este curso no le corresponde, al menos no como tema principal, discutir cómo cada persona tiene almacenada en su cerebro esa gramática ni cómo esta les permite a las personas mantener, reproducir y cambiar sus relaciones interpersonales.

Dado el modelo de estudios de la facultad de Estudios Generales Letras, cualquiera de estos cursos debería ser a la vez una invitación a la lingüística en general para alguien que nunca la ha estudiado. Del hecho de que ustedes se hayan sentido adecuadamente 'invitados' o no dependerá el que ese último objetivo se haya logrado.

\section{¿Para qué un curso de gramática?}

Una de las primeras cosas que debería llamarnos la atención es si estudiar gramática vale la pena dentro de la formación general. Durante la edad media, la 'Grammatica' era un curso obligatorio de formación general... Pero, en primer lugar, ya pasó mucho tiempo desde entonces, nuestro conocimiento sobre el mundo ha mejorado notablemente y es discutible que la formación de los monjes europeos medievales tenga por qué parecerse a la de los profesionales peruanos de hoy (o, por lo menos, en qué aspectos sí y en cuáles no). En segundo lugar, esos cursos medievales eran sobre todo para que los monjes aprendieran una lengua diferente de su lengua materna (el latín); no eran cursos sobre cómo son las lenguas. En contraste, en el curso que hemos compartido

4 Estos son las tres opciones de cursos que tienen los estudiantes de Estudios Generales Letras para cursar en su paso por esta facultad. En el caso de Teoría General del Lenguaje y Estructura del Lenguaje, los profesores tienen la posibilidad de brindar un distinto enfoque del curso. 
y en cualquier otro curso análogo sabemos que los alumnos ya saben una o más lenguas (en este caso, que ya saben castellano), y que la mayoría de ustedes no viene a clase a aprender ningún idioma. De hecho, cuando Luis Jaime Cisneros, un notable profesor de lingüística de nuestra universidad, empezó a dictar en los primeros ciclos del pregrado creó cursos de Lengua que reemplazaran a unos viejos cursos de español, ya que él no entendía qué razón podría haber para enseñar estos últimos a hispanohablantes. En tercer lugar, cuando se enseñaba gramática en la formación general medieval había un claro fin práctico: se asumía que el estudiante obtendría las reglas para producir textos orales y escritos considerados correctos y para evitar errores (es decir, formas consideradas incorrectas). Este aspecto práctico-prescriptivo no forma parte, para nada, del núcleo de la lingüística; y lo que se enseña en un primer curso de gramática es cómo (parece que) hablan los hablantes (del castellano o de cualquier lengua), no cómo deberían hablar. Así que ese fin práctico inmediato desaparece.

En el número del año pasado de la revista institucional de Estudios Generales Letras, Miguel Rodríguez-Mondoñedo, profesor lingüista de nuestra universidad y del curso de Estructura, ofrece tres respuestas de por qué valdría la pena un curso de gramática. A su juicio, el fin práctico-instrumental se mantiene en la medida en que es necesario saber algo de gramática para poder desempeñarse adecuadamente en el castellano escrito formal (como el que se usa en las universidades y los ámbitos profesionales). Pero a la vez habría una cualidad propedéutica especial de los cursos de lingüística para enseñar cómo se construyen las hipótesis y las teorías científicas ${ }^{5}$. A ello Rodríguez-Mondoñedo añade el hecho de que el lenguaje es un rasgo particularmente importante y peculiar del ser humano que merece ser estudiado y entendido ${ }^{6}$. Hasta aquí las respuestas de Miguel Rodríguez-Mondoñedo (2015).

Yo creo que los lingüistas no han hecho lo suficiente para demostrar que los cursos de gramática son útiles. Pongo un ejemplo. Creo que es reconocida

5 Miguel Rodríguez-Mondoñedo es uno de los lingüistas que están especialmente orgullosos de los logros y descubrimientos hechos por su disciplina, a tal punto de considerarlos un buen modelo para otras disciplinas.

6 Quizá este último punto funcione mejor para justificar que exista el curso de Teoría General del Lenguaje, más que para hacerlo con el de Estructura. 
la utilidad que estudiar lógica puede tener: la lógica es una disciplina formal que evalúa argumentos; bien enseñada, puede ser útil para mejorar la calidad de cualquier argumentación y de cualquier discusión (sin necesidad alguna de usar simbolitos); a la par, conocer el sistema de la lógica simbólica de primer orden facilita manejarnos y desenvolvernos en varias otras disciplinas que la usan y que son a su vez útiles para muchas cosas (la filosofía, la matemática, la estadística, la informática... iy la lingüística!). En todas las disciplinas hay cuestiones lingüísticas; pero no siempre queda claro que una formación básica en lingüística o en gramática pueda ayudar a resolverlas.

Sí es cierto que el estudio del léxico y la gramática forma parte del núcleo de la lingüística; y que si cualquiera quiere estudiar o practicar cualquier otro campo dentro de la lingüística tendrá que saber lo suficiente de gramática.

\section{¿De qué ideas espero que el curso los haya convencido?}

Hay, para empezar, un par de datos terminológicos que a menudo las personas desconocían antes de haber llevado el curso. No se trata de ideas importantes en sí, pero no estar al tanto de estos datos puede generar confusiones que sí son importantes en discusiones en distintos ámbitos. Los siguientes párrafos, sobre los términos 'lenguaje’, 'idioma', 'lengua' y ‘variedad', puede resultar demasiado enciclopédica, por eso la coloco en un tamaño de fuente menor.

Los lingüistas suelen hablar de el lenguaje. Con 'el lenguaje', se suelen referir a una capacidad subyacente que permite a las personas producir y entender enunciados en un idioma, y les permite utilizarlo para hacer diversas cosas. El lenguaje, entonces, subyace a todos los idiomas. En la vida cotidiana, las personas suelen hablar, en cambio, de distintos 'lenguajes'. Los lingüistas son los personajes raros que se apartan de la manera en que la mayoría de castellanohablantes usa esa palabra. Así, en los textos técnicos de lingüística nunca se dice que ningún idioma sea un lenguaje (nunca se diría 'el lenguaje shipibo', p.e.), sino que más bien se dice que cada idioma es una manifestación de el lenguaje. 
Hay un montón de otras cosas a las que llamamos 'lenguajes' y de las que todos hablamos todo el tiempo (p.e. se dice 'el lenguaje de la danza', 'el lenguaje de las emociones', 'el lenguaje de los astros', 'el lenguaje de las películas de Wes Anderson', 'el lenguaje de los gorriones', 'el lenguaje de la extrema derecha', 'el lenguaje no verbal'). En estos casos, 'lenguaje' sirve para referir a objetos de naturaleza muy distinta, pero ninguno de esos objetos es el lenguaje. Como recomendación general, lean con atención y revisen con cuidado en cada caso a qué se refiere una determinada persona al decir 'un lenguaje' o 'el lenguaje', sobre todo si se trata de algún filósofo, un crítico cultural o un científico social.

El genérico para entidades tales como el castellano, el danés, el quechua, el shipibo o el swahili, que no se ve afectado por el problema terminológico anterior es 'idioma(s)'. También pueden usarse sin riesgo de confusión 'lengua(s)' y, más técnicamente, la frase 'lengua(s) histórica(s)'. De esa manera, el castellano, el danés, el quechua, el shipibo y el suajili son cada uno un idioma, una lengua y una lengua histórica. Términos como 'habla' y 'dialecto' son intrínsecamente vagos y a menudo están cargados de valoraciones sociales. El término comodín, valorativamente neutro, usado por los lingüistas para denotar cualquier manera de hablar parecido entre los miembros de un grupo de personas es 'variedad(es)'. Hasta aquí el resumen terminológico que, debo resaltar, no es una cita.

Ahora lo que voy a hacer es listar un conjunto de ideas que espero que el curso los haya hecho notar o de las que espero que los hayamos convencido. Son muy generales, pero, a mi juicio, si se acuerdan de esas ideas de aquí a cinco años este curso habrá valido la pena.

- El lenguaje y las lenguas cumplen varias y distintas funciones, i.e. sirven para muchas cosas. Una de las más generales y fundamentales es representar situaciones, otra es transmitir información. Pero también sirven para saludar, pedir cosas, mostrar afecto o para 'trollear'... 
- Para que una palabra exista lo único que se requiere es que las personas las usen. 'Suculino', 'holi' y 'yolo' indudablemente existen.

- Las personas hablan el mismo idioma de maneras muy diferentes entre sí. Eso no es nada malo y, al contrario, es bastante interesante.

- Los límites de las lenguas históricas nunca están bien definidos. Ello es así justamente porque las personas hablan de distintas maneras entre sí. Siempre ha habido diversidad.

- Todas las lenguas humanas que existen o han existido han tenido estructura y han sido útiles para sus hablantes.

- Lo anterior es cierto independientemente de si alguna vez tal lengua tuvo o no escritura. Las letras, las tildes, la ortografía y la escritura no son, en sentido estricto, parte del lenguaje.

- Los hablantes a menudo no son conscientes ni pueden dar cuenta verbal de todas las propiedades acústicas, semánticas y gramaticales de las palabras, las oraciones y las otras unidades lingüísticas. El estudio de estas distintas propiedades requiere rigurosidad y, a menudo, una cierta jerga técnica.

Ninguna de las ideas listadas se refiere a cómo es la estructura de las lenguas y podrían muy bien cualquiera de ellas discutirse en los cursos de Teoría General del Lenguaje o Lenguaje y Sociedad.

\section{¿Qué hemos hecho (y qué no) en este curso?}

Alguna vez escuché de Jorge Iván Pérez, el profesor de esta comisión del curso de Estructura, la mejor justificación para que el curso se llame como se llama: a saber, las lenguas tienen estructura en la medida en que tienen elementos (unidades lingüísticas) que a su vez tienen relaciones entre sí. Toda oración, por ejemplo, es una unidad lingüística; pero, a la vez, toda oración contiene -está formada por- monemas (otras unidades lingüísticas). Igualmente, la frase 'mi mamá' es un constituyente de la frase 'para mi mamá'.

Respecto de cómo están estructuradas las lenguas, entonces, creo que el curso ha bastado para sostener que (i) toda lengua tiene unidades (fonemas, monemas, palabras, frases, oraciones) que están en relación con otras unida- 
des del mismo o de diferente tipo; que (ii) los signos simples (monemas) y los signos compuestos (oraciones) tienen una forma sonora ${ }^{7} \mathrm{y}$ un significado; $\mathrm{y}$ que (iii) las unidades lingüísticas y sus reglas de combinación pertenecen a cierto componente o subsistema (fonológico, léxico, morfológico y sintáctico). Esta última idea es también la que guía la manera en la que los gramáticos suelen estructurar sus gramáticas descriptivas (los textos que describen la estructura de una cierta lengua). Como prueba, menciono aquí un par de índices de dos gramáticas escritas en castellano:

Índice de la Nueva gramática básica de la lengua española (2011)

1. Cuestiones generales

2. Fonética y fonología

3. Morfología

4. Sintaxis

4.1. Clases de palabras y sus grupos sintácticos

4.2 Las funciones

4.3 Las construcciones sintácticas fundamentales

Índice de Una gramática del wãrnsöjöt (puinave) (2008)

I. Reseña histórica y cultural sobre el pueblo puinave y el wãrnsöjöt yedojert (lengua puinave)

II. Fonología

III. Morfología

IV. Sintaxis y tipos de oración

V. Comparación del wãrnsöjöt con otras lenguas del noroccidente amazónico

A esas tres ideas muy generales hay que añadir otras dos muy importantes. La primera es respecto del significado de los signos compuestos (palabras y oraciones). En todas las lenguas, en mayor o menor medida, el significado de las palabras y oraciones se "construye" o "articula" a partir del significado de sus partes. A esta propiedad de los signos compuestos se le llama 'composicio-

7 Esta formulación tiene el problema de excluir a las lenguas de señas. Quizá sería mejor decir 'forma sensorial'. 
nalidad'. Se dice que el significado de las palabras compuestas y de las oraciones es composicional.

La segunda idea es la de la doble articulación de las lenguas. Si nuestros signos proposicionales son compuestos es porque están articulados a partir de signos simples. Esto trae una ventaja combinatoria, porque a partir del número grande pero finito de elementos léxicos (signos simples) se pueden producir infinitos signos proposicionales. Ahora, como sabemos, cada signo simple es a la vez una cadena de elementos aún más simples sin significado (fonemas); lo cual permite que a partir de un reducido número de elementos mínimos (una lengua cualquiera suele tener entre 12 y 35 fonemas) se construya un número enormemente grande de elementos léxicos. Esta propiedad de economía combinatoria es esencial a la estructura de las lenguas humanas, y es por esta propiedad que a partir de un número finito de elementos se puedan producir potencialmente infinitos enunciados.

Ahora bien, creo que vale la pena volver a la sumilla preparada por Jorge para el sílabo:

Plan de curso de Estructura del lenguaje

El curso les muestra a los alumnos cómo se puede estudiar el lenguaje científicamente. Les ofrece la oportunidad de examinar la estructura y el funcionamiento del lenguaje humano a partir de la observación de su uso como sistema formal de representación y comunicación. Se muestra, en primer lugar, que toda lengua histórica está compuesta por diversas variedades - todas ellas legítimas desde el punto de vista de la Lingüística- que son abstracciones de los conocimientos individuales de los hablantes, de modo que la estructura del lenguaje se encuentra, en última instancia, en el sistema cognitivo que subyace a la actuación lingüística de los usuarios de una lengua. Posteriormente, se examinan los distintos componentes (o subsistemas) que conforman el conocimiento lingüístico, mostrando sus elementos y su organización interna, así como su interacción (Pérez Silva 2016).

Y creo que vale la pena porque ese pequeño párrafo es especialmente explícito no solo en lo que pretendíamos abordar, sino en ciertas asunciones o premisas que hemos asumido aquí sin discutirlas mucho. 
- La primera premisa que hemos asumido es que la gramática puede entenderse como un estudio 'científico' en el mismo sentido en que son científicas la ciencia física y la ciencia económica. Ello supone que tenemos ciertos datos (cómo hablan las personas, qué enunciados producen; $\mathrm{y}$, cuáles les parecen aceptables y cuáles no) que buscamos explicar mediante la postulación de reglas subyacentes. Las reglas gramaticales se entienden entonces no como reglas prescriptivas de conducta, sino como hipótesis para explicar los datos.

- La segunda premisa es que este conjunto de reglas que explican la conducta lingüística tienen un correlato cognitivo. Dicho de otra manera, la idea es que todo hablante de una lengua tiene en su cabeza una "gramática mental" y, en ese sentido, saber una lengua es tener un cierto "conocimiento". Saber alemán y saber yagua es, de acuerdo con este enfoque, tener almacenados en la mente un conjunto de elementos (un léxico y una gramática). Ello implica que toda hipótesis de reglas gramaticales será una hipótesis sobre cómo procesa y funciona la mente de la gente.

- Una tercera premisa, que presupone la anterior, es que la vaguedad en los límites de los idiomas o lenguas históricas se explica porque todo idioma no es más que una abstracción de los conocimientos individuales de sus hablantes.

- Finalmente, hay algo que no está del todo explícito en la sumilla pero que es crucial para entender esta propuesta de curso: hemos hecho énfasis en que, si bien el lenguaje sirve para comunicarnos y para representar situaciones, no todos los elementos de una lengua cumplen una función comunicativa o una función de representación. Hay elementos que son puramente arbitrarios (como el hecho de que 'silla' o 'leche' sean palabras femeninas). Y hay elementos (o tipos de unidades) que, aunque sí cumplen funciones comunicativas no se definen ni por su significado ni por su funcionalidad comunicativa sino en términos estrictamente formales (por sus propiedades estructurales y/o combinatorias; esto vale para categorías gramaticales como la de sustantivo o verbo, y para relaciones estructurales como la de sujeto y objeto). 
Hay, por supuesto, muchos temas relevantes que no han sido abordados. No hemos profundizado en cómo están organizados los sonidos de las lenguas, por ejemplo. Eso no está mal. Algo que sí me apena que no hayamos hecho un poco más es precisar qué lenguas además del castellano o el quechua hay en el país y cómo son estas. Sobre ello, les recomiendo un libro escrito para estudiantes de secundaria por Luis Andrade y el mismo Jorge Iván Pérez (2009), y la clase magistral de Roberto Zariquiey para Aula Abierta (2014).

Un comentario final sobre los 'arbolitos'. Como sabrán, a menudo Estructura es conocido como "el curso en que se hacen arbolitos" (diagramas arbóreos que describen la estructura de palabras, frases u oraciones). Y, como notarán, en ese curso solo hemos hecho diagramas arbóreos en la última sesión de clase de la última semana de clases. Eso no me parece mal. Hay, asimismo, muchas versiones del curso que hacen muchos 'arbolitos'. Eso tampoco está mal. Pero siempre debe quedar claro que ni los arbolitos son parte de la estructura de las lenguas (son meras herramientas gráficas para dar cuenta de esta) ni usarlos es indispensable para un curso de gramática. 


\section{Referencias bibliográficas}

Asociación de Academias de la Lengua Española (AALE) y Real Academia Española (RAE). Nueva gramática básica de la lengua española, Salvador Gutiérrez (responsable). Obra basada en la Nueva gramática de la lengua española publicada el año anterior. Espasa, 2011.

Andrade Ciudad, Luis y Jorge Iván Pérez Silva. Las lenguas del Perú. PUCP (Oficina Central de Admisión), 2009.

Girón, Jesús Mario. Una gramática del wãrnsöjöt (puinave). Traducción de su tesis doctoral defendida ante la Vrije Universiteit. Landelijke Onderzoekschool Taalwetenschap (the Netherlands National Graduate School of Linguistics, LOT), 2008. lotpublications.nl/Documents/185_fulltext.pdf.

Pérez Silva, Jorge. Sílabo del curso de Estructura del Lenguaje. PUCP (Estudios Generales Letras), 2016.

Rodríguez-Mondoñedo, Miguel."El lenguaje y los Estudios Generales.” Mural de Letras, año 10, número 15, 2015, p. 25. facultad.pucp.edu.pe/generales-letras/wp-content/ uploads/2016/o7/Mural-de-Letras-2015-PARA-WE B.pdf

Zariquiey, Roberto. "La diversidad lingüística en el Perú”. YouTube, PUCP (Estudios Generales Letras), 31 de oct. 2014, youtube.com/watch?v=5pgDtrlP2Ws. 